\title{
CONTROLE DA TOLERÂNCIA A GLICOSE NA NUTRIÇÃO PARENTERAL
}

\author{
Ana Maria Kazue Miyadahira* \\ Carmen Lúcia Sfair** \\ Maria Sumie Koizumi*** \\ Mieko Ide Fujimoto**** \\ Joel Faintuch *****
}

MIYADAHIRA, A. M. K.; SFAIR, C. L.; KOIZUMI, M. S.; FUJIMOTO, M. I.; FAINTUCH, J. Controle da tolerância à glicose na nutrição parenteral. Rev. Esc. Enf. USP, São Paulo, 14(1):21-28, 1980.

Foram estudados prospectivamente 10 pacientes cirúrgicos não diabéticos, submetidos a hiperalimentação parenteral, no sentido de se determinar a incidência de intolerância d glicose durante este tratamento. Adicionalmente, compararam-se métolos de vigilancia do metabolismo glicídico, a saber: glicosúria e glicemia, estimadas com fitas reagentes, $e$ glicemia fornecida pelo "Auto-Analyser", com o propósito de se estabelecer qual destes seria mais apropriada para uso rotineiro. As conclusões foram: 1) A incidência de intolerância à glicose, classificada com um ou mais episódios de glicosúria positiva, ou de glicemia superior a $180 \mathrm{mg} / 100 \mathrm{ml}$, foi de $60 \%$ aos doentes no primeiro dia, e de $80 \%$ na duração total do tratamento; 2) $A$ intolerância à glicose foi muito mais freqüentemente detectada pela glicosúria (com fita) do que pelo "Dextrostix" ou pela glicemia do "Auto Analyser, pois a glicosúria foi pesquisada com freqüencia muito maior. Conclui-se que, embora a glicosúria possa refletir adequadamente o metabolismo da glicose em tolas as circunstâncias, é o melhor exame para vigilância básica dos casos de nutrição parenteral, desde que a função renal dos pacientes seja satisfatória, devido ao seu baixo custo e facilidade de utilização.

\section{INTRODUÇĀO}

A hiperalimentação parenteral, método terapêutico introduzido por DUDRICK et alii ${ }^{4}$, a partir de 1968 , constitui modalidade altamente eficaz de reabilitação nutricional para doentes criticamente enfermos.

A assistência contínua necessária para o sucesso do tratamento exige a presença de uma equipe multiprofissional especializada composta de médicos, enfermeiros, bioquímicos e farmacêuticos, trabalhando em perfeito entrosamento.

Ao enfermeiro cabe atuar junto ao paciente desde a instalação até o término desta terapêutica. É da sua responsabilidade orientar o paciente quanto ao tratamento a que será submetido, assistí-lo durante a introdução, manutenção e retirada do catéter, controlar a infusão da solução e detectar precocemente as possíveis complicações ${ }^{8}$.

Dentre os controles preconizados para detectar complicações, destacam-se as medidas de vigilância da glicemia e da glicosúria, tendo-se em vista as elevadas doses de glicose administradas rotineiramente a esses pacientes ${ }^{3}$.

* Auxiliar de Ensino da disciplina Enfermagem Médico-Cirúrglca I da EEUSP.

* Enfermeira Chefe da 2.a Clínica Cirúrgica do Hospital das Clínicas da FMUSP.

*** Professor Assistente da disciplia Enfermagem Médico-Cirúrgica I da EEUSP. Mestre em Enfermagem.

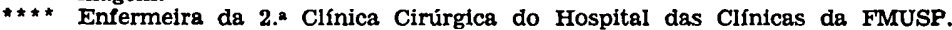

$\star \star \star \star \star$ Professor Assistente do Departamento de Cirurgia da FMUSP. 
Para PHILLIPS 12 os mais fáceis indicadores de tolerância à glicose são os testes urinários para detectar a glicose e corpas cetônicos. Alguns autores são de opinião que esses exames devem ser realizados rotineiramente de $6 \mathrm{em} 6$ horas ${ }^{1,6,8}$, 9,12,14, ou de 4 em 4 horas ou cada 2 horas ${ }^{10}$.

Em texto de enfermagem escrito em nosso meio, GOMES et alii ${ }^{7}$, é preconizado que cabe ao enfermeiro controlar a glicosúria a cada miç̧ão do paciente e, se ele estiver com catéter vesical, esse controle deve ser feito a cada duas horas, no início do esquema; quando o tratamento se prolonga por semanas, o controle pode ser efetuado com menor freqüência, pois, há tendência à estabilização nos níveis de tolerância à glicose.

Há, ainda, recomendação geral de que a glicemia seja controlada antes do início e durante todo o tratamento do paciente.

Observa-se, entretanto, que as rotinas estabelecidas, nem sempre são concordantes, no que diz respeito ao esquema de vigilância da tolerância à glicose nesses pacientes.

Desta forma, com os objetivos de estabelecer a resposta glicêmica à nutrição parenteral em nosso meio, e de pôr em evidência as vantagens e os inconvenientes dos vários métodos para supervisão da glicemia e da glicosúria comumente utilizados, os autores realizaram um estudo prospectivo da glicemia e da glicosúria em 10 pacientes adultos, portadores de afecções cirúrgicas, que se submeteram ao tratamento em apreço.

\section{CASUISTICA E MÉTODO}

Foram avaliados, consecutivamente, 10 pacientes, submetidos a nutrição parenteral internados em uma unidade cirúrgica de um hospital estatal, a partir de janeiro de 1979, os quais foram selecionados unicamente em função das seguintes especificações: 1) não portador de insuficiência renal ou hepática significativa, ou seja, recebendo a mistura padrão de Glicose a $50 \%$ e Aminoácidos preparada pela farmácia central do hospital ${ }^{5}$; 2 ) não portador de diabetes mellitus; 3 ) de idade inferior a 70 anos; 4) e jejum absoluto, nutrindo-se exclusivamente pela via venosa.

Os métodos escolhidos para a rerificação da resposta metabólica foram: a glicosúria e a glicemia, estimadas por fitas reagentes (respectivamente "L-combur-5test", Laboratório Boehringer, São Paulo, e "Dextrostix", Laboratório Ames, São Paulo); bem como a glicemia convencional: ("Auto-Analyser", laboratório central do hospital em questão.

0 estudo foi feito segundo o seguinte protocolo: 1) no primeiro dia tanto ia glicemia ("Dextrostix") como a glicosúria ("Combur-test") eram registradas de 3 em 3 horas; 2) no período subseqüente, até o término da nutrição parenteral a glicosúria ("Combur-test") era registrada às miç̧ões ou de $6 \mathrm{em} 6$ horas nos pacientes com sonda vesical, conjugada a glicemia ("Dextrostix") uma vez ao dia, às 8 horas da manhã; 3 ) adicionalmente, era colhida glicemia antes do início e durante o tratamento, na proporção de 2 a 5 vezes por semana, de acordo com a evolução; 4) informações gerais - Todos os dados anteriores foram interpretados à luz 
da resposta terapêutica dos pacientes, assim como do aparecimento de complicações, tais como febre e infecção, intervenção cirúrgica e outras intercorrências.

\section{RESULTADOS}

1) Informações gerais - A idade média dos 10 enfermos estudados foi dé 51,1 anos (20 a 68 anos), sendo que oito dos dez pacientes tinham mais de 40 anos. O sexo masculino predominou na proporção de 7:3.

Os diagnósticos de entrada de seis enfermos eram moléstias neoplásicas (cinco ıumores gástricos, um tumor de esôfago); os quatro outros eram casos de colangite aguda, fístula gástrica (ulcera gástrica operada), abcesso retroperitonial pós adrenalectomia bilateral (moléstia de Cushing) e estenose péptica do esôfago.

A duração média ıda nutrição parenteral foi de 14,5 dias ( 3 a 4,0 dias). Três pacientes foram tratados por periodos de até uma semana, outros três permaneceram com alimentação intravenosa por uma a duas semanas, três foram nutridos por 15-30 dias, e o último caso restante por 40 dias. A indicação terapêutica mais freqüente nesta casuística foi preparo pré-operatório ( 6 pacientes); nos demais a justificativa do tratamento foi suporte pós-cirúrgico ( 2 casos), abcesso retroperitonial (1 caso) e fístula gástrica ( 1 caso). Apenas dois pacientes desta série não foram operados durante sua internação; dos oito restantes, seis foram submetidos a apenas uma intervenção cirúrgica e dois, a um total de três operações cada.

A curva de temperatura esteve sempre normal em seis doentes (cinco em preparo pré-operatório, e um já submetido a cirurgia); os demais exibiram um ou mais picos febris no decurso do estudo.

Houve três óbitos nesta série, todos ocasionados pela moléstia original; nos demais casos, interrompeu-se o aporte parenteral quando o paciente foi julgado suficientemente melhorado para se submeter ao tratamento cirúrgico (três casos), ou para receber alta ou transferência para outro serviço (quatro enfermos).

2) Glicemias laboratoriais - A glicemia pré-alimentação parenteral, colhida geralmente 24, horas antes da instalação do regime, acha-se descrita na Tabela 1 . Pode-se notar que, embora o protocolo excluisse diabéticos ou quadros equivalentes (havia um caso de moléstia de Cushing, porém já corrigido cirurgicamente, e recebendo apenas doses de manutenção de corticosteróides), houve elevada incidência de glicemias anormais neste levantamento, somente em parte explicáveis pela infusão contínua de soro glicosado isotônico que os pacientes recebiam nesta fase.

TABELA 1

Glicemia anterior à nutrição parenteral

\begin{tabular}{cc}
\hline Valor & Freqüuéncia \\
\hline $70-100 \mathrm{mg} / 100 \mathrm{ml}$ & 3 \\
$100-180 \mathrm{mg} / 100 \mathrm{ml}$ & 3 \\
Acima de $180 \mathrm{mg} / 100 \mathrm{ml}$ & 4 \\
\hline
\end{tabular}

* máximo de $225 \mathrm{mg} / 100 \mathrm{ml}$

As glicemias anotadas ao longo da terapêutica calórico-protéica têm seu comportamento especificado na Tabela 2. Embora a maioria dos valores tenha sido distribuída numa faixa considerada normal, vários valores deslocaram-se em certas 
ocasiōes para a chamada zona "cirúrgica" da glicemia (13), tida como aceitável apenas por periodos limitados.

\section{TABELA 2}

Glicemia durante a realizaçäo do tratametno

\begin{tabular}{lc}
\hline Valor & Frequiência \\
\hline Abaixo de $50 \mathrm{mg} / 100 \mathrm{ml}$ & 0 \\
de $50-180 \mathrm{mg} / 100 \mathrm{ml}$ & 6 \\
Acima ${ }^{\star}$ de $180 \mathrm{mg} / 100 \mathrm{ml}$ & 3 \\
Não verificado & 1 \\
\hline
\end{tabular}

* máximo de $328 \mathrm{mg} / 100 \mathrm{ml}$

3) Glicemia e glicosúria com fitas reagentes - primeiro dia. - À evolução da glicosúria nas horas iniciais foi documentado nas tabelas 3 e 4 , enquanto a de glicemia ("Dextrostix"), em igual periodo, pode ser vista nas Tabelas 5 e 6 . Chama a atenção o maior número de glicosúrias suspeitas que de glicemias anormais, porém, o início da instalação destes desvios foi variável, de menos de 6 até mais de 12 horas após a introdução da mistura nutritiva.

TABELA 3

Glicosúria no primeiro dia

\begin{tabular}{cc}
\hline Valor & Frequiência \\
\hline Sempre $(-)$ & 4 \\
+ até ++ & 4 \\
Mais de ++ & 2 \\
\hline
\end{tabular}

TABELA 4

Início da glicosúria

\begin{tabular}{lc}
\hline \multicolumn{1}{c}{ Período } & Frequência \\
\hline $0-6 \mathrm{~h}$ & 1 \\
$0-12 \mathrm{~h}$ & 1 \\
Após $12 \mathrm{~h}$ & 4 \\
\hline
\end{tabular}

TABELA 5

Glicemia (Dextrostix) no primciro dia

\begin{tabular}{lc}
\hline Valor & Frequiência \\
\hline $50-130$ & 7 \\
$175-250$ & 3 \\
\hline
\end{tabular}

TABELA 6

Infcio da hiperglicemia (Dextrostix) acima de $175 \mathrm{mg} / 100 \mathrm{ml}$

\begin{tabular}{lc}
\hline Periodo & Frequiência \\
\hline $0-6 \mathrm{~h}$ & 2 \\
$6-12 \mathrm{~h}$ & 1 \\
\hline
\end{tabular}

4) Glicemia e glicosúria (fita) no decurso do tratamento - Estes achados podem ser acompanhados no Quadro 1. Também aqui foi mais comum o encontro 
de glicosúrias anormais, que de glicemias medidas pelo "Dextrostix", ou mesmo pelo laboratório, que se situassem acima do limiar mais freqüente de excreção renal de glicose, da ordem de $180 \mathrm{mg} / 100 \mathrm{ml}$.

QUADRO 1

Correlaçåo entre a glicemia e a glicosúria durante a nutrição parenteral, diagnosticadas por diferentes métodos

\begin{tabular}{|c|c|c|c|}
\hline Casos & $\begin{array}{c}\text { Glicosúria } \\
\text { (Combur-test) }\end{array}$ & $\begin{array}{c}\text { Glicemia } \\
\text { (Dextrostix) }\end{array}$ & $\begin{array}{c}\text { Glicemia } \\
\text { (Auto-analyser) }\end{array}$ \\
\hline $\begin{array}{l}2 \\
1 \\
5 \\
2\end{array}$ & $\begin{array}{l}\text { sempre (-) } \\
-1+ \\
-1++ \\
-1+++\end{array}$ & $\begin{array}{r}45-130 \\
45-150 \\
45-175 \\
\text { (1) } 90-130 \\
\text { (1) } 45-250\end{array}$ & $\begin{array}{l}\text { abaixo de } 180 \mathrm{mg} / 100 \mathrm{ml} \\
\text { abaixo de } 180 \mathrm{mg} / 100 \mathrm{ml} \\
3 \text { casos }<180 ; 2 \text { casos }>180 \\
1 \text { caso }<180 ; 1 \text { caso }>180 ;\end{array}$ \\
\hline
\end{tabular}

\section{COMENTÁRIOS}

A série de casos aqui estudada representa uma parcela razoavelmetne típica de pacientes cirúrgicos que necessitam de nutrição parenteral: pacientes de meia idade ou mais velhos, frequentemente portadores de enfermidades neoplásicas, e submetidos a intervençōes cirúrgicas não raramente complicadas ou múltiplas. É sob este prisma, de desnutrição prolongada e estresse cirúrgico significativo, que interpretamos a incidência relativamente elevada de intolerância à sobrecarga de glicose, anotada em nossos pacientes, apesar da exclusão de diabéticos conhecidos. A gravidade do comprometimento sistêmico destes enfermos pode ser aferida também pela apreciável mortalidade observada $(3 / 10$ ou $30 \%)$, devida, entretanto, às enfermidades de base, pré-existentes (não houve complicações significativas da hiperalimentação parenteral nesta série).

A possibilidade de instalação de hiperglicemia, nas primeiras horas de introdução da nutrição intravenosa foi estudada por SANDERSON \& DEITEL ${ }^{16}$.

$\mathrm{Na}$ experiência destes autores, a uma infusão constante de glicose, na dose de $400 \mathrm{mg} / \mathrm{min}$ (equivalente a $576 \mathrm{~g}$ de glicose nas 24 horas, ou pouco mais de dois litros da mistura usual de hiperalimentação parenteral), seguia-se hiperglicemia e hiperinsulinemia, com picos de 1-2 horas para a glicose e de 2-3 horas para a insulina. Estes parâmetros estabilizavam antes de decorridas 6 horas, em níveis pouco aumentados de $120-140 \mathrm{mg} / 100 \mathrm{ml}$ para a glicemia e taxas variáveis para a insulinemia.

Prolongando-se a infusão para um período de 7 dias, notaram aqueles investigadores que a glicemia se manteve dentro de valores pouco acima do normal, graças a um eficiente aujste na liberação da insulina endógena, mesmo quando a oferta de glicose era sucessivamente elevada para $450 \mathrm{mg} / \mathrm{min}$ e depois pra $500 \mathrm{mg}$ / min (equivalente a $770 \mathrm{~g}$ de glicose/dia, ou três litros de mistura parenteral). Não houve diurese osmótica ou hiperglicemia que necessitasse de suplementação exógena da insulina, em nenhuma ocasião, e à interrupção da prescrição nutritiva não se seguiu hipoglicemia reacional.

$\mathrm{Na}$ comparação das respostas observadas nestes casos com aquelas obtidas no presente trabalho, deve-se ressalvar que, embora as doses iniciais de glicose administradas por SANDERSON \& DEITEL ${ }^{16}$ tenham sido maiores que as aqui em- 
pregadas (média de $2000 \mathrm{ml}$ de mistura nutritiva nas primeiras 24 horas, versus, apenas $1000 \mathrm{ml} /$ dia em nossos doentes), os resultados não são equivalentes, pois, a homeostare glicídica perfeita por ele relatada dizia respeito a pacientes selecionados, relativamente jovens e em bom estado geral. No presente estudo os pacientes não foram selecionados, ou melhor ainda, foram selecionados usualmente em função das suas más condições metabólicas, fruto de desnutrição avançada, neoplasias malignas, idade avançada, e não raramente processos infecciosos de apreciável gravidade. Nestas circunstâncias, foi observada intolerância à glicose em diferentes etapas do tratamento, sem preferência nítida pelas primeiras 6 ou 12 horas de infusão; houve glicosúrias positivas nas primeiras horas em $60 \%$ dos casos e, nos dias subseqüentes, em $80 \%$ dos doentes.

É preciso enfatizar que a grande maioria destas glicosúrias, da ordem de apenas + a ++ , corresponde a espoliações de não mais que $0,5 \%$ a $1,0 \%$ da glicose infundida; e que, mesmo com glicosúrias máximas, de ++++ , freqüentemente a excreção urinária é de apenas $2 \%$ da glicose em circulação ${ }^{2}$. Perdas desta ordem, especialmente de curta duração, como foi o caso dos enfermos estudados, não acarretam qualquer peerturbação clínica, e poderiam inclusive não ser corrigidas com insulina suplementar, embora seja rotina a prescrição de doses progressivas de insulina simples nestes casos 5,15 .

No que concerne à eficácia de cada uma das medidas diagnósticas, no sentido de melhor espelhar o aparecimento de intolerância à glicose, foi verificado que, durante a utilização da nutrição intravenosa, apenas 3 dentre 10 casos, com determinações regulares da glicemia laboratorial, e um dentre $10 \mathrm{com}$ vigilância da glicemia pelo "Dextrostix", apresentaram resultados superiores a $180 \mathrm{mg} / 100 \mathrm{ml}$, valor considerado como limiar para aparecimento de glicosúria positiva.

Outrossim, sendo a enfermeira o elemento da equipe de saúde que permanece quase permanentemente junto ao pacinte e, também, a responsável por este controle, sugere-se que investigações ulteriores sejam feitas no sentido de se estabelecer qual a periodicidade ideal para esta avaliação.

Esta conclusão seria aparentemente um contrasenso, porquanto os testes urinários com fita apresentam um erro intrínseco de $20-30 \%{ }^{13}$, da mesma ordem daquele observado com as fitas para glicemia ${ }^{2}$ (o "Destrostix" torna-se ainda menos sensível em faixas de glicemia muito alta), e somente a glicemia laboratorial pode ser aceita como estimativa padrão; cabe assinalar, entretanto, que, neste estudo, somente a glicosúria foi realizada de $6 \mathrm{em} 6$ horas; a glicemia dada pela fita reagente era colhida apenas uma vez ao dia e a glicemia "Auto-Analyser", de 2 a 5 vezes por semana. Dada a natureza eminentemente transitória da glicosúria na nutrição parenteral de pacientes não diabéticos, como foi o presente caso, o teste mais sensivel para o registro destas flutuações ocasionais passaria a ser aquele mais assiduamente realizado, ainda que de precisão possivelmente inferior.

As fitas reagentes são sensiveis à luz, umidade e seus reagentes facilmente podem evaporar. Para seu uso efetivo, sugere-se que, além de obedecer o período de validade da fita e precaução de não tocar com os dedos os locais dos reagentes, a fita deve ser testada antes em pacientes com glicosúria positiva.

Embora o controle de glicosúria com fitas reagentes seja o exame mais prático e confortável para a vigilância da tolerância à glicose, não se exclui a necessi- 
dade de outros exames mais sensiveis. Assim, na suspeita de um episódio hipoglicêmico, as fitas reativas para glicosúria tornam-se inúteis. É necessário controlar a glicemia seja por fitas reativas ou por métodos laboratoriais. Igualmente, a glicosúria é inútil naqueles casos onde a excreãço de glicose pela urina passa a se efetuar de forma aleatória e imprevisivel, como acontece nas lesões renais apreciáveis.

Por outro lado, na suspeita de episódios de hiperglicemia, a glicemia por métodos laboratoriais torna-se imprescindível porque há necessidade de análise quantitativa precisa. Isto vem demonstrar que, mesmo na avaliação de um parâmetro como a tolerância à glicose, é importante a existência de uma equipe multiprofissional entrosada e que todos estejam atentos para uma avaliação global do paciente.

Nesta série de pacientes cirúrgicos, controlados predominantemente pela glicosúria, não ocorreu caso algum de hiperglicemia superior a $400 \mathrm{mg} / 100 \mathrm{ml}$, ou de hipoglicemia inferior a $50 \mathrm{mg} / 100 \mathrm{ml}$. Todos os pacientes puderam receber o volume programado de dieta intravenosa, embora isto não tenha obrigatoriamente propiciado o sucesso terapêutico, devido à extrema gravidade de alguns casos.

\section{Agradecimentos}

Os autores expressam os seus agradecimentos à Maria Cristina O. Souza, Maria Helena Maret, Sebastião Fernandes e a todos que colaboraram neste trabalho.

MIYADAHIRA, A. M. K.; SFAIR, C. L.; KOIZUMI, M. S.; FUJIMOTO, M. I.; FAINTUCH, J. Intolerance glucose control in the parenteral hyperalimentation. Rev. Esc. Enf. USP, São Paulo, 14(1):21-28, 1980.

Ten non-diabetic surgical patients, submitted to parenteral hyperalimentation, were prospectively studied, in order to determine the incidence of glucose intolerance during this treatment. Additionally three methods for the control of glucose metabolism, namely glucosuria and glycemia, given by reagent strips, and standard blood sugar, determined at the auto-analyser, were compared, with the purpose of establishing which of these tests is best suited for routine utilization. It was concluded that: 1) The incidence of glucose intoleran$c e$, defined as one or more episodes of positive glucosuria, or of blood sugar higher than $180 \mathrm{mg} / 100 \mathrm{ml}$, was $60 \%$ in the first day of intravenous nutrition, and $80 \%$ if the whole period of treatment; 2) Glucose intolerance was more often detected by urine testing than by blood determinations, either with Dextrositx or at the a auto-analyser as the glucosuria test: was performed much more frequently; 3) It is concluded that although the findings of tests for glucosuria may not adequately reflect glucose metabolism in all circumstances, they represent the choicesexamination for basic monitoring of patients receiving intravenous hyperalimentation, as soon as renal function is satisfactory, because of its low expense and its casy application.

\section{REFERENCIAS BIBLIOGRAFICAS}

1. BIEBUYCK, J. F. The hyperalimentation unit: the collaboration of clinician, biochemist, pharmacist and nurse. S. Afr. Med. J., Africa, 50: 1724-5, Oct. 1976.

2. DAVIDOFF, F. Rational metabolic management of diabetic patients undergoing surgery. In: SKILLMAN, J. J., ed. Intensive care, Boston, Little Brown, 1975, p. 353-85.

3. DUDRICK, S. J.; MaCFADYEN, B. V.; VAN BUREN, C. T.; RUBERG, R. L.; MAYNARD, A. T. Parenteral hyperalimentation, metabolis problems and solutions. Ann. Surg., Philadelphia, 176: $259,1972$.

4. DUDRICK, S. J.; WILMORE, D. W.; VARS, H. M.; RHOADS, J. E. Long-term total parenteral nutrition with growth, development and positive nitrogen balance. Surgery, St. Louis, 64: 136, 1968.

5. FAINTUCH, J. Alimentação parenteral prolongada: adultos. In: RAIA, A. A. Manual do pré e pós-operatório. São Paulo, Manole,, 1978, p. 769-78.

6. Listematização técnica da alimentação parenteral. In: FAINTUCH, I.; MACHADO, M. C. C.; WRETLIND, A.; JACOBSON, S.; WESTER, P. O.; SOLASSOL, C.; JOYEUX, H. Allmentação parenteral prolongada. São Paulo, Manole, 1976, p. 301-10. 
7. GOMES, A. M.; OLIVEIRA, C.; KAMADA, C.; KIMURA, M. Nutrição parenteral prolongada. In: Enfermagem na unidade de terapia intensiva. São Paulo, E. P. U. e EDUSP, 1978, p. 119-30.

8. GRIGGS, B. A. TPN nursing. In: DUDRICK, S. J., ed. Clinical parenteral nutrition. New York, McGraw Laboratories, p. 32-7.

9. LAMY, P. P. Total parenteral nutrition: guidelines and preceutions. Hosp. Formul., Chicago, 11: 414-6, Aug. 1976

10. LINS, L. C. S. Participação da enfermagem na hiperalimentação parenteral. A enfermagem atual, Rio de Janeiro, 1(1): 5-20, 1978.

11. MACEDO, H. Nutrição parenteral. Enfermagem: o jornal brasileiro de enfermagem, Rio de Janeiro, 2(25): 6, abr. 1979 .

12. PHILLIPS, K. J. Cuidados de enfermagem na nutrição parenteral total. In: FISCHER, J. E. Natrição parenteral. Rio de Janeiro, Guanabara Koogan, 1978, p. 96-105.

13. ROSSINI ,A. A. \& HARE, J. W. How to control the bloob glucose level in the surgical diabetic patient. Arch Aurg., Chicago, 111: 945, 1976.

14. RUBENSTEIN, R. B. \& WOODS, D. Hyperalimentation: your role in an innovative procedure. J. Pract. Nurs., New York, 27(5): 26.9, May, 1977.

15. RYAN JUNIOR, J. A. Complications of total parenteral nutrition. In: FISCHER, J. E., ed. Total parenteral nutrition. Boston, Little, Brown, 1976, p. 55-100.

16. SANDERSON, I. \& DEITEL, M. The response in patients undergoing prolonged intravenous hyperalimentation. Surgical Forum, Philadelphia, 23: 64, 1972. 